\title{
Clonal relatedness of Proteus mirabilis strains causing urinary tract infections in companion animals and humans
}

\author{
Cátia Marques $^{\mathrm{a}}$, Adriana Belas ${ }^{\mathrm{a}}$, Catarina Aboim ${ }^{\mathrm{a}}$, Graça Trigueiro ${ }^{\mathrm{b}}$, Patrícia Cavaco-Silva ${ }^{\mathrm{c}, \mathrm{d}}$, \\ Luís Telo Gama ${ }^{\mathrm{a}}$, Constança Pomba ${ }^{\mathrm{a}, *}$ \\ ${ }^{a}$ Interdisciplinary Centre of Research in Animal Health, Faculty of Veterinary Medicine, University of Lisbon, Avenida da Universidade Técnica, 1300-477, Lisboa, Portugal \\ ${ }^{\mathrm{b}}$ Laboratório de Análises Clínicas Dr. Joaquim Chaves, Av. General Norton de Matos, 71 R/C, 1495-148 Algés, Portugal \\ ${ }^{\mathrm{c}}$ Centro de Investigação Interdisciplinar Egas Moniz, Instituto Universitário Egas Moniz, Campus Universitário, Quinta da Granja, Monte de Caparica, 2829-511, Caparica, \\ Portugal \\ ${ }^{\mathrm{d}}$ Technophage, S.A., Avenida Prof. Egas Moniz, 1600-190, Lisboa, Portugal
}

\section{A R T I C L E I N F O}

\section{Keywords:}

Proteus mirabilis

Urinary tract infection

Companion animal

Human

Clonal relatedness

\begin{abstract}
A B S T R A C T
Proteus mirabilis is a major cause of urinary tract infection (UTI) in humans and companion animals. This study aimed to evaluate the antimicrobial resistance, virulence and clonal relatedness of $P$. mirabilis isolated from dogs, cats and humans with UTI.

P. mirabilis isolated from companion animals $(\mathrm{N}=107)$ and humans $(\mathrm{N}=76)$ with UTI were compared by PFGE analysis after overnight NotI macro-restriction using Dice/UPGMA with a 1.5\% tolerance. Strains were characterized for antimicrobial resistance by disk diffusion. Twenty-four resistance genes and four virulence genes were screened by PCR.

Thirty-nine clusters (similarity $>80 \%$ ) and 73 single pulse-types were detected. Nine clusters included $P$. mirabilis isolated from community and hospital patients, including strains with $100 \%$ similarity. A high number of clusters $(43.6 \%, n=17 / 39)$ included strains from companion animals and humans. Similarity between some companion animal and human strains varied between $80-100 \%$. One strain from a dog was $100 \%$ similar to one human community-acquired $P$. mirabilis. One $P$. mirabilis from a cat was found to be $94.7 \%$ and $92.4 \%$ similar to community and hospital patient strains, respectively. $P$. mirabilis CMY-2-producers did not cluster all together. Nevertheless, cluster C36 included five $P$. mirabilis from companion animals (similarity 85.8\%-95.7\%), of which, four (80\%) were multidrug-resistant CMY-2-producers.

This study shows that companion animals and humans become infected with closely related $P$. mirabilis strains. The high number of clusters containing companion animals and human strains points to the zoonotic nature of $P$. mirabilis. These results underline the potential role of companion animals as reservoirs and in the dissemination of uropathogenic $P$. mirabilis to humans and vice versa.
\end{abstract}

\section{Introduction}

Proteus mirabilis are known to cause community-acquired urinary tract infections (UTI) in humans and are important agents of nosocomial UTIs (Armbruster and Mobley, 2012; Costa et al., 2018). After E. coli, which is the main cause of UTI, $P$. mirabilis is the second most common Enterobacteriacea isolated in companion animals with UTI (Moyaert et al., 2017). The increasing antimicrobial resistance trends in bacteria isolated from companion animals creates concerns regarding the role of companion animals as reservoirs of resistant bacteria to humans (Marques et al., 2017; Pomba et al., 2017). CMY-2-producing $P$. mirabilis, which are resistant to third-generation cephalosporins and frequently multidrug-resistant (MDR), are increasingly being reported in companion animals in Portugal (Marques et al., 2017). Since companion animals with UTI caused by $P$. mirabilis have high loads of bacteria in urine and faeces (Gaastra et al., 1996), the risk of dissemination of uropathogenic $P$. mirabilis, including potential resistant strains, into the owners living environment may be an important hazard.

Little is known about the clonal relatedness between human and companion animal uropathogenic $P$. mirabilis. Yet, this information is relevant to understand the role of companion animals and human as reservoirs of $P$. mirabilis. Only a limited number of European studies on resistance monitoring of UTI in companion animals are available

\footnotetext{
* Corresponding author at: Faculdade de Medicina Veterinária, Universidade de Lisboa, Av. da Universidade Técnica, 1300-477, Lisboa, Portugal.

E-mail address: cpomba@fmv.ulisboa.pt (C. Pomba).
} 
(Marques et al., 2017; Moyaert et al., 2017). Furthermore, only early studies report the frequency of virulence factors among $P$. mirabilis from companion animals (Bijlsma et al., 1995; Gaastra et al., 1996).

The main goal of this study was to determine the clonal relatedness of $P$. mirabilis isolated from companion animals (dogs and cats) and humans with UTI. Additionally, these uropathogenic $P$. mirabilis were characterised for the presence of antimicrobial resistance and virulence genes.

\section{Material and methods}

\subsection{Bacteria isolates}

This study included $P$. mirabilis isolated from humans with UTI $(\mathrm{N}=76)$ at a private clinical analysis laboratory $(\mathrm{N}=50)$ in 2014 and between 2006-2015 from hospital microbiology laboratories $(\mathrm{N}=26)$ collecting samples from patients in the Lisbon area. The $P$. mirabilis isolated at the human private laboratory were obtained from humans with community-acquired UTI (CA-human). Samples from the hospital laboratories were obtained from hospital patients with UTI (HAhuman) and were likely hospital-acquired. However, it was not possible to ensure that these infections were nosocomial due to insufficient patient data. Non-duplicate $P$. mirabilis strains from companion animals with UTI (dogs $N=94$, cats $N=13$ ) were obtained from 1999 to early 2015 at the Laboratory of Antimicrobial Resistance from the Veterinary Teaching Hospital of the Faculty of Veterinary Medicine/University of Lisbon and therefore all companion animals were also from the Lisbon area.

Besides being collected from the same geographical region, there were no direct epidemiological relations between the sampled humans and companion animals. It was also not possible to obtain information regarding the overall daily contact of the sampled humans and companion animals with UTI. Moreover, data on prior antimicrobial use was not available.

\subsection{Bacteriological methods}

P. mirabilis strains were isolated by standard quantitative urine cultures and species identification was conducted by phenotypical tests (API20E and VITEKII, BioMérieux, Marcy-l'Étoile, France) and confirmed by P. mirabilis species-specific PCR (Marques et al., 2017).

\subsection{Susceptibility testing}

Antimicrobial susceptibility testing was performed by disk diffusion method according to CLSI guidelines (CLSI, 2018, 2017). Isolates were tested for susceptibility against a total of twenty-six antimicrobials

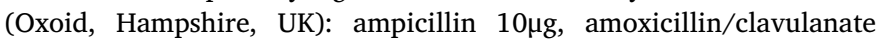
$30 \mu \mathrm{g}$, cefoxitin $30 \mu \mathrm{g}$, cefotaxime $30 \mu \mathrm{g}$, ceftazidime $30 \mu \mathrm{g}$, cefovecin $30 \mu \mathrm{g}$, piperacillin-tazobactam $110 \mu \mathrm{g}$, cefepime $30 \mu \mathrm{g}$, imipenem $10 \mu \mathrm{g}$, meropenem $10 \mu \mathrm{g}$, aztreonam $30 \mu \mathrm{g}$, ertapenem $10 \mu \mathrm{g}$, nalidixic acid $30 \mu \mathrm{g}$, ciprofloxacin $5 \mu \mathrm{g}$, enrofloxacin $5 \mu \mathrm{g}$, norfloxacin $10 \mu \mathrm{g}$, levofloxacin $5 \mu \mathrm{g}$, gentamicin $10 \mu \mathrm{g}$, kanamycin $30 \mu \mathrm{g}$, netilmicin $30 \mu \mathrm{g}$, to-

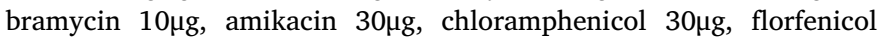
$30 \mu \mathrm{g}$, fosfomycin $50 \mu \mathrm{g}$ and trimethoprim/sulfamethoxazole $25 \mu \mathrm{g}$.

For cefovecin and enrofloxacin results interpretation, the veterinary CLSI breakpoints were applied (CLSI, 2018). Florfenicol (a fluorinated compound in use for bovine respiratory infections) was tested for epidemiological purposes only. Since there are no defined florfenicol breakpoints for companion animals nor humans, interpretation diameter limits were derived from the veterinary CLSI breakpoints for swine infections (CLSI, 2018). The Societé Française de Microbiology breakpoints were used for fosfomycin (SFM, 2010). Since gentamicin breakpoints only vary between veterinary and human CLSI guidelines by $1 \mathrm{~mm}$ in the susceptible/intermediate categorisation (CLSI, 2017, 2018), human CLSI was used. The remaining antimicrobials were interpreted according to human CLSI breakpoints (CLSI, 2017), since breakpoints either are equal or are not determined in veterinary CLSI standards.

Antimicrobial categories were used to characterize MDR Enterobacteriacea (Magiorakos et al., 2012). P. mirabilis were considered as MDR when resistant to three or more antimicrobial categories.

\subsection{Antimicrobial resistance determinants}

Ampicillin resistant/intermediate isolates were tested for the presence of $b l a_{\mathrm{TEM}}, b l a_{\mathrm{SHV}}$ and $b l a_{\mathrm{OXA}-1}$ beta-lactamase genes by PCR (Pomba et al., 2006). The presence of ESBL (bla $\left.a_{\mathrm{CTX}-\mathrm{M} \text {-type }}\right)$ and AmpC (bla $a_{\mathrm{CMY}-\mathrm{type}}, b l a_{\mathrm{LAT}-\mathrm{type}}, b l a_{\mathrm{BIT}-1}, b l a_{\mathrm{DHA}-\mathrm{type}}, b l a_{\mathrm{MOX}-\mathrm{type}}, b l a_{\mathrm{ACT}-\mathrm{type}}, b l a_{\mathrm{FOX}}$ type and $\left.b a_{\text {MIR-type }}\right)$ beta-lactamase genes among third-generation cephalosporin resistant strains has been characterized elsewhere (Marques et al., 2017).

Antimicrobial resistant/intermediate isolates were further screened by PCR for the presence of antimicrobial resistance genes against aminoglycosides [aphAI-IAB, $\operatorname{aac}\left(6^{\prime}\right)-I b$ and $\left.a a c\left(3^{\prime}\right)-I V\right]$ (Frana et al., 2001; Sáenz et al., 2004; Robicsek et al., 2006a), phenicols (floR) (Sáenz et al., 2004), fluoroquinolones ( $q n r \mathrm{~A}, q n r \mathrm{~B}, q n r C$, $q n r D$ and $q n r S$ ) (Robicsek et al., 2006b; Cavaco et al., 2009; Wang et al., 2009) and trimethoprim-sulfamethoxazole (sul1, sul2, sul3, dfrA12 and dfrIa [targeting dfrA1, dfrA5, dfrA15, dfrA15b, dfrA16, dfrA16b]) (Sáenz et al., 2004; Guerra et al., 2001; Maynard et al., 2003; Perreten and Boerlin, 2003).

\subsection{Virulence genes}

All $P$. mirabilis were screened by PCR for the presence of $u c a A$ (urothelial cell adhesion fimbriae [UCA/NAF]), $m r p A$ (mannose-resistant Proteus-like fimbriae [MR/P]) and pmfA (Proteus mirabilis fimbriae [PMF]) fimbriae genes and for the presence of haemolysin $\mathrm{HpmA} / \mathrm{HpmB}$ codifying genes (Sosa et al., 2006; Cestari et al., 2013).

\subsection{Population structure and data analysis}

P. mirabilis were characterized by overnight restriction with NotI (Thermo Scientific ${ }^{\mathrm{TM}}$, Lisbon, Portugal) and subsequent PFGE on a CHEF DR II-apparatus (Biorad, Hercules, CA, USA). The restriction fragments were resolved in a $1 \%$ agarose gel (agarose pulse-field grade, Nzytech Genes and Enzymes, Lisbon, Portugal) using the following previously described block conditions: $1-30 \mathrm{~s}$ for $8 \mathrm{~h}$ followed by $30-70 \mathrm{~s}$ for $16 \mathrm{~h}$ at $14{ }^{\circ} \mathrm{C}, 6 \mathrm{~V} / \mathrm{cm}^{2}$ (Sabbuba et al., 2003).

P. mirabilis NotI restriction PFGE-pattern analysis was conducted with the Bionumerics software, version 6.6, (Applied Maths from BioMérieux, Marcy-l'Étoile, France) using Dice/ UPGMA clustering method with a tolerance of $1.5 \%$ and a clustering cut-off of $80 \%$.

The SAS statistical software package for Windows, version 9.3, (SAS Institute Inc, Cary, North Carolina, USA) was used to perform statistical analysis. Comparisons between groups were conducted by Fisher's Exact test, with an alpha value of 0.05 .

\section{Results}

\subsection{Antimicrobial resistance and antimicrobial resistance determinants}

Considering beta-lactam non-susceptible strains (categorized as antimicrobial resistant or intermediate isolates), $P$. mirabilis from companion animals and CA-humans were frequently resistant only to ampicillin (46.9\%, $\mathrm{N}=15 / 32$ and $86.7 \%, \mathrm{~N}=13 / 15$, respectively) while HA-human $P$. mirabilis were frequently resistant to ampicillin and intermediate or resistant to amoxicillin/clavulanate $(61.5 \%, \mathrm{~N}=8 / 13)$ (Table 1).

Beta-lactam non-susceptible $P$. mirabilis strains frequently 
Table 1

Antimicrobial resistance frequencies of companion animal and human $P$. mirabilis strains.

\begin{tabular}{|c|c|c|c|c|c|c|}
\hline \multirow[t]{2}{*}{ Antimicrobials } & \multicolumn{2}{|c|}{$\begin{array}{l}\text { Companion animal } \\
(\mathrm{N}=107)\end{array}$} & \multicolumn{2}{|l|}{$\begin{array}{l}\text { CA-human } \\
(\mathrm{N}=50)\end{array}$} & \multicolumn{2}{|l|}{$\begin{array}{l}\text { HA-human } \\
(\mathrm{N}=26)\end{array}$} \\
\hline & $\% \mathrm{R}$ & $\% \mathrm{I}$ & $\% \mathrm{R}$ & $\% \mathrm{I}$ & $\% \mathrm{R}$ & $\% \mathrm{I}$ \\
\hline Ampicillin & $28.0 \%(\mathrm{n}=30)$ & $1.9 \%(n=2)$ & $30.0 \%(\mathrm{n}=15)$ & $0 \%(\mathrm{n}=0)$ & $50.0 \%(\mathrm{n}=13)$ & $0 \%(\mathrm{n}=0)$ \\
\hline Amoxicillin/clavulanate & $9.4 \%(\mathrm{n}=10)$ & $5.6 \%(n=6)$ & $2.0 \%(\mathrm{n}=1)$ & $2.0 \%(\mathrm{n}=1)$ & $3.8 \%(\mathrm{n}=1)$ & $26.9 \%(\mathrm{n}=7)$ \\
\hline Cefoxitin & $9.4 \%(n=10)$ & $0 \%(\mathrm{n}=0)$ & $0 \%(\mathrm{n}=0)$ & $0 \%(\mathrm{n}=0)$ & $0 \%(\mathrm{n}=0)$ & $0 \%(\mathrm{n}=0)$ \\
\hline Cefotaxime & $9.4 \%(n=10)$ & $0 \%(\mathrm{n}=0)$ & $0 \%(n=0)$ & $0 \%(\mathrm{n}=0)$ & $0 \%(\mathrm{n}=0)$ & $0 \%(\mathrm{n}=0)$ \\
\hline Ceftazidime & $5.6 \%(n=6)$ & $3.7 \%(n=4)$ & $0 \%(\mathrm{n}=0)$ & $0 \%(\mathrm{n}=0)$ & $0 \%(\mathrm{n}=0)$ & $0 \%(n=0)$ \\
\hline Cefovecin & $9.4 \%(n=10)$ & $0 \%(\mathrm{n}=0)$ & $0 \%(\mathrm{n}=0)$ & $0 \%(\mathrm{n}=0)$ & $0 \%(\mathrm{n}=0)$ & $0 \%(n=0)$ \\
\hline Piperacillin-tazobactam & $1.9 \%(\mathrm{n}=2)$ & $0 \%(\mathrm{n}=0)$ & $0 \%(\mathrm{n}=0)$ & $0 \%(\mathrm{n}=0)$ & $0 \%(\mathrm{n}=0)$ & $0 \%(\mathrm{n}=0)$ \\
\hline Cefepime & $0 \%(\mathrm{n}=0)$ & $5.6 \%(\mathrm{n}=6)$ & $0 \%(n=0)$ & $0 \%(\mathrm{n}=0)$ & $0 \%(\mathrm{n}=0)$ & $0 \%(\mathrm{n}=0)$ \\
\hline Imipenem & $0 \%(\mathrm{n}=0)$ & $7.5 \%(\mathrm{n}=8)$ & $0 \%(n=0)$ & $2 \%(\mathrm{n}=1)$ & $0 \%(\mathrm{n}=0)$ & $0 \%(\mathrm{n}=0)$ \\
\hline Meropenem & $0 \%(\mathrm{n}=0)$ & $0 \%(\mathrm{n}=0)$ & $0 \%(\mathrm{n}=0)$ & $0 \%(\mathrm{n}=0)$ & $0 \%(\mathrm{n}=0)$ & $0 \%(\mathrm{n}=0)$ \\
\hline Aztreonam & $1.9 \%(\mathrm{n}=2)$ & $0 \%(n=0)$ & $0 \%(\mathrm{n}=0)$ & $0 \%(\mathrm{n}=0)$ & $0 \%(\mathrm{n}=0)$ & $0 \%(n=0)$ \\
\hline Ertapenem & $0 \%(\mathrm{n}=0)$ & $0 \%(\mathrm{n}=0)$ & $0 \%(\mathrm{n}=0)$ & $0 \%(\mathrm{n}=0)$ & $0 \%(\mathrm{n}=0)$ & $0 \%(\mathrm{n}=0)$ \\
\hline Nalidixic acid & $26.2 \%(\mathrm{n}=28)$ & $4.7 \%(n=5)$ & $16.0 \%(\mathrm{n}=8)$ & $4.0 \%(\mathrm{n}=2)$ & $42.3 \%(\mathrm{n}=11)$ & $0 \%(\mathrm{n}=0)$ \\
\hline Ciprofloxacin & $8.4 \%(n=9)$ & $5.6 \%(\mathrm{n}=6)$ & $8.0 \%(n=4)$ & $2.0 \%(n=1)$ & $26.9 \%(n=7)$ & $3.8 \%(\mathrm{n}=1)$ \\
\hline Enrofloxacin & $26.2 \%(\mathrm{n}=28)$ & $1.9 \%(\mathrm{n}=2)$ & $16.0 \%(\mathrm{n}=8)$ & $0 \%(\mathrm{n}=0)$ & $42.3 \%(\mathrm{n}=11)$ & $0 \%(\mathrm{n}=0)$ \\
\hline Norfloxacin & $5.6 \%(\mathrm{n}=6)$ & $1.9 \%(\mathrm{n}=2)$ & $6.0 \%(n=3)$ & $0 \%(\mathrm{n}=0)$ & $19.2 \%(\mathrm{n}=5)$ & $3.8 \%(\mathrm{n}=1)$ \\
\hline Levofloxacin & $7.5 \%(\mathrm{n}=8)$ & $0 \%(\mathrm{n}=0)$ & $8.0 \%(n=4)$ & $0 \%(\mathrm{n}=0)$ & $23.1 \%(\mathrm{n}=6)$ & $0 \%(\mathrm{n}=0)$ \\
\hline Gentamicin & $6.5 \%(n=7)$ & $2.8 \%(\mathrm{n}=3)$ & $10.0 \%(n=5)$ & $0 \%(\mathrm{n}=0)$ & $30.8 \%(n=8)$ & $0 \%(n=0)$ \\
\hline Kanamycin & $20.6 \%(n=22)$ & $5.6 \%(\mathrm{n}=6)$ & $12.0 \%(\mathrm{n}=6)$ & $2.0 \%(\mathrm{n}=1)$ & $11.5 \%(\mathrm{n}=3)$ & $0 \%(\mathrm{n}=0)$ \\
\hline Netilmicin & $3.7 \%(n=4)$ & $2.8 \%(\mathrm{n}=3)$ & $2.0 \%(\mathrm{n}=1)$ & $0 \%(\mathrm{n}=0)$ & $7.7 \%(\mathrm{n}=2)$ & $0 \%(\mathrm{n}=0)$ \\
\hline Tobramycin & $5.6 \%(\mathrm{n}=6)$ & $1.9 \%(\mathrm{n}=2)$ & $4.0 \%(n=2)$ & $0 \%(\mathrm{n}=0)$ & $0 \%(\mathrm{n}=0)$ & $0 \%(\mathrm{n}=0)$ \\
\hline Amikacin & $2.8 \%(n=3)$ & $0.9 \%(n=1)$ & $0 \%(\mathrm{n}=0)$ & $0 \%(\mathrm{n}=0)$ & $0 \%(\mathrm{n}=0)$ & $0 \%(\mathrm{n}=0)$ \\
\hline Trimethoprim/sulfamethoxazole & $35.5 \%(n=38)$ & $1.9 \%(\mathrm{n}=2)$ & $26.0 \%(\mathrm{n}=13)$ & $0 \%(\mathrm{n}=0)$ & $38.5 \%(\mathrm{n}=10)$ & $3.8 \%(\mathrm{n}=1)$ \\
\hline Chloramphenicol & $28.0 \%(n=30)$ & $18.7 \%(n=20)$ & $20.0 \%(\mathrm{n}=10)$ & $8.0 \%(n=4)$ & $11.5 \%(\mathrm{n}=3)$ & $11.5 \%(n=3)$ \\
\hline Florfenicol & $5.6 \%(\mathrm{n}=6)$ & $10.3 \%(n=11)$ & $4.0 \%(n=2)$ & $2.0 \%(\mathrm{n}=1)$ & $0 \%(\mathrm{n}=0)$ & $15.4 \%(n=4)$ \\
\hline Fosfomycin & $5.6 \%(\mathrm{n}=6)$ & $0 \%(n=0)$ & $2.0 \%(\mathrm{n}=1)$ & $0 \%(\mathrm{n}=0)$ & $0 \%(\mathrm{n}=0)$ & $0 \%(\mathrm{n}=0)$ \\
\hline
\end{tabular}

Legend: N, total sample number; \%R, percentage of resistant isolates; \%I percentage of intermediate isolates; n, number of isolates; HA-human, human hospitalpatient UTI; CA-human, human community-acquired UTI.

Table 2

Antimicrobial resistance genes detected in antimicrobial non-susceptible $P$. mirabilis strains.

\begin{tabular}{|c|c|c|c|c|c|c|}
\hline Antimicrobial & Gene & Overall & Companion animal & CA-human & HA-human & $\mathrm{P}_{\text {value }}{ }^{\mathrm{a}}$ \\
\hline Beta-lactams & $b l a_{\mathrm{TEM}}$ & $\begin{array}{l}88.3 \% \\
(n=53 / 60)\end{array}$ & $\begin{array}{l}84.4 \% \\
(n=27 / 32)\end{array}$ & $\begin{array}{l}86.7 \% \\
(n=13 / 15)\end{array}$ & $\begin{array}{l}100 \% \\
(n=13 / 13)\end{array}$ & 0.4320 \\
\hline \multirow[t]{2}{*}{ Third-generation cephalosporins } & $b l a_{\mathrm{CMY}-2}$ & $\begin{array}{l}90.0 \% \\
(n=9 / 10)\end{array}$ & $\begin{array}{l}90.0 \% \\
(n=9 / 10)\end{array}$ & - & - & - \\
\hline & $b l a_{\text {DHA-1 }}$ & $\begin{array}{l}10.0 \% \\
(\mathrm{n}=1 / 10)\end{array}$ & $\begin{array}{l}10.0 \% \\
(\mathrm{n}=1 / 10)\end{array}$ & - & - & - \\
\hline Fluoroquinolones & $q n r D$ & $\begin{array}{l}16.4 \% \\
(n=9 / 55)\end{array}$ & $\begin{array}{l}17.6 \% \\
(n=6 / 34)\end{array}$ & $\begin{array}{l}30 \% \\
(n=3 / 10)\end{array}$ & $\begin{array}{l}0 \% \\
(\mathrm{n}=0 / 11)\end{array}$ & 1 \\
\hline \multirow[t]{2}{*}{ Aminoglycosides } & $a p h A I-I A B$ & $\begin{array}{l}56.2 \% \\
(n=27 / 48)\end{array}$ & $\begin{array}{l}65.5 \% \\
(n=19 / 29)\end{array}$ & $\begin{array}{l}55.6 \% \\
(\mathrm{n}=5 / 9)\end{array}$ & $\begin{array}{l}30.0 \% \\
(\mathrm{n}=3 / 10)\end{array}$ & 0.1426 \\
\hline & $\operatorname{aac}\left(3^{\prime}\right)-I V$ & $\begin{array}{l}2.1 \% \\
(n=1 / 48)\end{array}$ & $\begin{array}{l}3.4 \% \\
(n=1 / 29)\end{array}$ & $\begin{array}{l}0 \% \\
(\mathrm{n}=0 / 9)\end{array}$ & $\begin{array}{l}0 \% \\
(\mathrm{n}=0 / 10)\end{array}$ & 1 \\
\hline \multirow[t]{4}{*}{ Folate pathway inhibitors } & sul1 & $\begin{array}{l}29.7 \% \\
(n=19 / 64)\end{array}$ & $\begin{array}{l}17.5 \% \\
(n=7 / 40)\end{array}$ & $\begin{array}{l}38.5 \% \\
(n=5 / 13)\end{array}$ & $\begin{array}{l}63.6 \% \\
(n=7 / 11)\end{array}$ & 0.0102 \\
\hline & sul2 & $\begin{array}{l}46.9 \% \\
(n=30 / 64)\end{array}$ & $\begin{array}{l}50.0 \% \\
(n=20 / 40)\end{array}$ & $\begin{array}{l}61.5 \% \\
(\mathrm{n}=8 / 13)\end{array}$ & $\begin{array}{l}18.2 \% \\
(\mathrm{n}=2 / 11)\end{array}$ & 0.6085 \\
\hline & sul3 & $\begin{array}{l}1.6 \% \\
(n=1 / 64)\end{array}$ & $\begin{array}{l}2.5 \% \\
(n=1 / 40)\end{array}$ & $\begin{array}{l}0 \% \\
(\mathrm{n}=0 / 13)\end{array}$ & $\begin{array}{l}0 \% \\
(\mathrm{n}=0 / 11)\end{array}$ & 1 \\
\hline & dfrIa & $\begin{array}{l}81.2 \% \\
(n=52 / 64)\end{array}$ & $\begin{array}{l}85.0 \% \\
(n=34 / 40)\end{array}$ & $\begin{array}{l}69.2 \% \\
(n=9 / 13)\end{array}$ & $\begin{array}{l}81.8 \% \\
(n=9 / 11)\end{array}$ & 0.3414 \\
\hline Phenicols & floR & $\begin{array}{l}6.8 \% \\
(n=5 / 74)\end{array}$ & $\begin{array}{l}9.8 \% \\
(n=5 / 51)\end{array}$ & $\begin{array}{l}0 \% \\
(\mathrm{n}=0 / 15)\end{array}$ & $\begin{array}{l}0 \% \\
(\mathrm{n}=0 / 8)\end{array}$ & 0.3161 \\
\hline
\end{tabular}

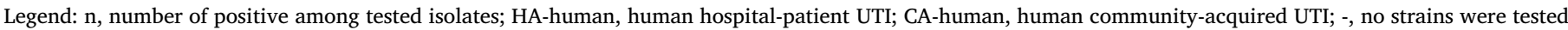

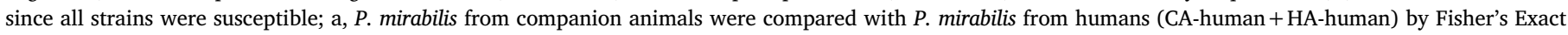
test.

harboured $b l a_{\mathrm{TEM}}(88.3 \%)$ while $b l a_{\mathrm{OXA}-1}$ and $b l a_{\mathrm{SHV}}$ were not detected (Table 2). Third-generation cephalosporin-resistance was only present in $P$. mirabilis from companion animals and was mostly linked to the presence of the $b l a_{\mathrm{CMY}-2}$ gene.

Resistance to fluoroquinolones was higher in isolates from HAhuman UTIs, followed by companion animal and CA-Human UTIs (Table 1). Three main fluoroquinolone-non-susceptible phenotypes were detected, namely non-susceptibility to nalidixic acid and enrofloxacin with or without ciprofloxacin-non-susceptibility and non-susceptibility to all tested fluoroquinolones including norfloxacin and levofloxacin. Interestingly, the non-susceptibility to nalidixic acid and enrofloxacin phenotype was significantly more common in companion animal $P$. mirabilis strains isolated prior to $2006(75 \%, \mathrm{~N}=12 / 16$, $\mathrm{P}=0.0003$ ) while non-susceptibility to all fluoroquinolones became 
significantly more frequent thereafter $(44.4 \%, \mathrm{~N}=8 / 18, \mathrm{P}=0.0031)$ (data not shown). Only $q n r D$ was detected in $P$. mirabilis from companion animals and humans (Table 2).

Among aminoglycosides, kanamycin and gentamicin showed the highest non-susceptibility frequencies (Table 1 ) and the aphAI-IAB was the most common resistance gene detected (Table 2).

Both in companion animal and human $P$. mirabilis, resistance against trimethoprim/sulfamethoxazole was high (26.0\%-38.5\%) (Table 1). The frequency of sul1 was significantly lower in $P$. mirabilis from companion animals when compared to strains from humans overall (Table 2). However, when compared to strains from CA-human only, a statistical significant difference was not detected $(P=0.1403)$. The frequency of sul2 in $P$. mirabilis from CA-human was significantly higher than in strains from HA-human $(P=0.0470)$. Nonetheless, sul1 and sul2 were detected in $P$. mirabilis from all patients while sul 3 was only detected in $P$. mirabilis from companion animals (Table 2). All trimethoprim/sulfamethoxazole non-susceptible $P$. mirabilis lacked $d f r A 12$ and several strains were negative for sul1, sul2 and sul3 (companion animal $37.5 \%, \mathrm{~N}=15 / 40$; CA-human $15.4 \%, \mathrm{~N}=2 / 13$; HA-human $18.2 \%, \mathrm{n}=2 / 11$ ). Overall, dfrIa was common in $P$. mirabilis from all patients (Table 2).

Chloramphenicol-resistance was high, especially in companion animal and CA-human P. mirabilis (Table 1). The floR gene was only detected in P. mirabilis from companion animals (Table 2) and all were coresistant to chloramphenicol and florfenicol.

MDR $P$. mirabilis were more frequent among HA-human $(42.3 \%$, $\mathrm{N}=11 / 26)$ strains, followed by companion animal $(21.5 \%, \mathrm{~N}=23$ / 107 ) and CA-human $(18 \%, N=9 / 50)$. However, most MDR P. mirabilis were only resistant to three antimicrobial categories (HA-human, $30.8 \%, \mathrm{~N}=8 / 26$; CA-human, $10 \%, \mathrm{~N}=5 \backslash 50$ and companion animals, $10.3 \%, \mathrm{~N}=11 / 107)$. $P$. mirabilis from human patients were resistant to up to 5 antimicrobial categories while third-generation cephalosporinresistant $P$. mirabilis from companion animals were resistant to 5 up to 9 antimicrobial categories.

\subsection{Virulence genes}

All fimbriae genes (ucaA, pmfA and $m r p A$ ) were highly frequent among $P$. mirabilis isolated from companion animals and humans (Table 3), nevertheless, ucaA gene was less frequent (Table 3). The haemolysin $H m p A / H m p B$ genes were present in all tested $P$. mirabilis (Table 3).

\subsection{Population structure}

Thirty-nine clusters and 73 unique pulse-types were detected (Supplementary Fig. 1). Nine clusters included $P$. mirabilis from both CA-human and HA-human UTIs (C2, C3, C8, C10, C13, C24, C26, C28 and C29) with some including highly related (Dice index $90 \%-100 \%$ ) CA-human and HA-human P. mirabilis strains (C2, C10, C13, C24, C26 and $\mathrm{C} 28$ ). It should be noted that some of these strains report to different time-periods and were obtained from unrelated patients.
A high number of clusters $(43.6 \% \mathrm{~N}=17 / 39)$ included $P$. mirabilis from companion animals and humans (C2, C3, C6, C13, C14, C15, C16, C17, C22, C25, C26, C27, C28, C31, C37, C38 and C39). The clonal relatedness between companion animal and human $P$. mirabilis varied between $80-100 \%$. Several companion animal strains included in these clusters were isolated from different time-periods. Cluster C28 is noteworthy since it includes one strain, from a dog, $100 \%$ similar to one CA-human $P$. mirabilis. Furthermore, one $P$. mirabilis from a cat (C2) was also found to be $94.7 \%$ and $92.4 \%$ similar to one CA-human and HAhuman strain, respectively. Some $P$. mirabilis strains isolated from dogs and cats (C21 and C34) also showed high similarity (90\%-100\%). Based on veterinary records, only strains FMV5954B/03 and FMV5955/ 03 were found to be epidemiologically related, since they were isolated in the same week from two cats admitted at the same veterinary practice.

P. mirabilis CMY-2-producer strains from companion animals did not cluster all together. However, C36 included five $P$. mirabilis from companion animals (85.8\%-95.7\% similarity), of which, four $(80 \%)$ were multidrug-resistant CMY-2-producers.

\section{Discussion}

Currently there are few studies characterizing the clonal population structure epidemiology of human or companion animal uropathogenic P. mirabilis strains (Gaastra et al., 1996; Sabbuba et al., 2003; Mathur et al., 2008). A high number of single pulse-types were detected both in human and in companion animal $P$. mirabilis strains in this study. Nevertheless, several PFGE clusters composed of $P$. mirabilis isolated in different years were detected, pointing to a higher dissemination of some clusters.

P. mirabilis is known to cause nosocomial infections (Armbruster and Mobley, 2012). The detection of several closely related HA-human strains likely resulted from nosocomial dissemination. The two identical $P$. mirabilis from two cats admitted at the same clinic, may also point to nosocomial events. Notably, the nine clusters that included $P$. mirabilis from CA-human and HA-human UTIs highlight the possible circulation of some strains between the hospital and community environment.

One previous study conducted on a limited number of $P$. mirabilis of several sources, detected one $P$. mirabilis strain from a dog that was closely related to one strain from a human (Schultz et al., 2015). In the present study, a high number of PFGE clusters (43.6\%) contained strains from companion animal and human origin, including some with significant similarity (90\%-100\%). Thus, these results point to the zoonotic potential of uropathogenic $P$. mirabilis. The close similarity of some strains from cats and dogs also highlights the possibility of $P$. mirabilis transfer between pets.

Overall, HA-human P. mirabilis seemed to have higher antimicrobial resistance frequencies. However, the fact that this study relied on convenience samples collected in different time-spans is a study limitation that restricts more extensive comparisons. Another limitation from this study was the lack of data regarding any prior antimicrobial treatment to the sampling.

Table 3

Companion animal and human P. mirabilis strains virulence genes.

\begin{tabular}{|c|c|c|c|c|c|}
\hline Virulence genes & $\begin{array}{l}\text { Overall } \\
(n=183)\end{array}$ & $\begin{array}{l}\text { Companion animal } \\
(n=107)\end{array}$ & $\begin{array}{l}\text { CA-human } \\
(n=50)\end{array}$ & $\begin{array}{l}\text { HA-human } \\
(n=26)\end{array}$ & $P$ value ${ }^{a}$ \\
\hline \multicolumn{6}{|l|}{ Fimbriae } \\
\hline$m r p A$ & $98.9 \%(n=181)$ & $99.1 \%(n=106)$ & $98 \%(n=49)$ & $100 \%(n=26)$ & 1 \\
\hline $\operatorname{pmfA}$ & $98.9 \%(n=181)$ & $100 \%(\mathrm{n}=107)$ & $96 \%(n=48)$ & $100 \%(n=26)$ & 0.1711 \\
\hline$u c a A$ & $85.2 \%(\mathrm{n}=156)$ & $86.9 \%(n=93)$ & $80 \%(n=40)$ & $88.5 \%(n=23)$ & 0.5272 \\
\hline \multicolumn{6}{|l|}{ Haemolysin } \\
\hline НтрА/НmpВ & $100 \%(\mathrm{n}=183)$ & $100 \%(\mathrm{n}=107)$ & $100 \%(\mathrm{n}=50)$ & $100 \%(\mathrm{n}=26)$ & 1 \\
\hline
\end{tabular}

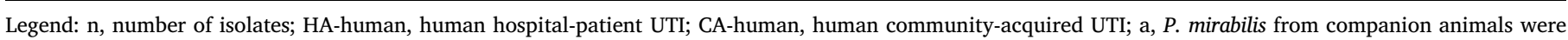
compared with $P$. mirabilis from humans (CA-human + HA-human) by Fisher's Exact test. 
Nonetheless, some of the higher resistance frequencies in this study were detected against important antimicrobials for UTI treatment, such as trimethoprim-sulfamethoxazole, fluoroquinolones and beta-lactams. A previous study from Portugal reported higher antimicrobial resistance frequencies among $P$. mirabilis from humans with communityacquired UTI (Costa et al., 2018). Part of the companion animal $P$. mirabilis strains here characterized have been included in a previous study analysing the temporal trends of antimicrobial resistance in UTI (Marques et al., 2017). It is noteworthy that in this previous study, a significant increase in MDR P. mirabilis third-generation cephalosporin resistance was detected due to the presence of CMY-2 beta-lactamase (Marques et al., 2017).

The $P$. mirabilis isolated from humans in this study were all thirdgeneration cephalosporin susceptible. However, the detection of AmpCproducing $P$. mirabilis from human infection is being increasingly reported in Europe (D'Andrea et al., 2011; Aogáin et al., 2016). Interestingly, in our study, several MDR CMY-2 producing $P$. mirabilis strains from companion animals, including unrelated strains from a wide timeframe, clustered together. This may point to the expansion of MDR CMY-2-producing PFGE C36 among companion animals. A previous study conducted in the UK, using whole genome sequencing (WGS), has reported the expansion of two clones of CMY-2-producing $P$. mirabilis in human infection (Aogáin et al., 2016). Since all CMY-2-producing $P$. mirabilis from companion animals from the present study were MDR and resistant to $5-9$ antimicrobial categories, their possible expansion is worrisome.

Companion animal and human $P$. mirabilis strains harboured similar antimicrobial resistance genes except bla $a_{\mathrm{CMY}-2}$, sul3, floR and aac(3)-IV, only detected in companion animals. Therefore, $P$. mirabilis from companion animals were reservoirs of important resistance genes. The high dissemination of sul1 and sul2 in P. mirabilis is in line with the high frequency of these resistance genes in other Enterobacteriaceae from Portugal (Antunes et al., 2005). The trimethoprim/sulfamethoxazole non-susceptible strains lacking sul1, sul2 and sul3 may harbour untested resistance mechanism such as the recently described sul4 (Razavi et al., 2017). The presence of $q n r D$ among fluoroquinolone-non-susceptible $P$. mirabilis agrees with previous published data (Harada et al., 2014; Jacoby et al., 2014; Schultz et al., 2017a; de Jong et al., 2018). Nevertheless, the frequency of $q n r D$ detected in P. mirabilis from companion animals in the present study was higher than in previous studies (Harada et al., 2014; Schultz et al., 2017a). The main acquired fluoroquinolone resistance mechanism in Enterobacteriaceae is the acquisition of mutations on the quinolone resistance-determining regions (QRDR) (Weigel et al., 2002; Harada et al., 2014). Furthermore, generally resistance to a given fluoroquinolone is accompanied by elevated non-wild type MIC values of other fluoroquinolones (Hernández et al., 2000). It was interesting to notice that several $P$. mirabilis strains from the present study did not have clinical cross-resistance to all tested fluoroquinolones. The higher clinical resistance of $P$. mirabilis towards enrofloxacin than to ciprofloxacin was also noted in a previous study conducted in P. mirabilis of dog origin and several QRDR mutations were reported (Harada et al., 2014). QRDR mutations of ciprofloxacinresistant and levofloxacin-susceptible $P$. mirabilis strains from humans have also been reported (Weigel et al., 2002). The temporal change in fluoroquinolone resistance phenotype in $P$. mirabilis from companion animals could be a consequence of antimicrobial use over time. The characterization of QRDR and determination of fluoroquinolone minimal inhibitory concentrations should be pursued in the future.

The aphAI-IAB aminoglycoside phosphotransferase has been previously detected in P. mirabilis through WGS (Di Pilato et al., 2016). To our knowledge, this is the first detection of aphAI-IAB in P. mirabilis from companion animals and the first report of its frequency among clinical relevant $P$. mirabilis. The detection of floR only in companion animal strains arguments to different chloramphenicol/florfenicol resistance epidemiology between companion animals and humans. In Salmonella, the floR is sometimes present in the Salmonella genomic island 1 (SGI1) (Hall, 2010). Interestingly, some SGI1 variants have already been described in MDR P. mirabilis from companion animals and humans (Schultz et al., 2015).

The high frequency of $u c a A, m r p A$ and $p m f A$ fimbriae genes detected in this study could be expected based on previous phenotypical and genotypic studies conducted in $P$. mirabilis from companion animals and humans, respectively (Bijlsma et al., 1995; Gaastra et al., 1996; Sosa et al., 2006). The comparison of UCA/NAF, MR/P and PMF fimbrial adhesins among strains from companion animals and humans in the future may help to better understand $P$. mirabilis zoonotic potential. Although, early phenotypical studies have shown that most $P$. mirabilis from dogs with UTI were haemolytic (Gaastra et al., 1996), to our knowledge, this is the first report of the haemolysin $H m p A / H m p B$ coding genes presence and frequency among companion animal strains. The high frequency of $H m p A / H m p B$ in $P$. mirabilis from humans agrees with previous results (Cestari et al., 2013).

There is a lack of studies comparing the clonal relatedness between $P$. mirabilis from companion animals and humans. Furthermore, the few existing studies are focused in selected resistant population from several infection sources (Schultz et al., 2015, 2017b). To our best knowledge, this is the first study comparing the clonal relatedness of a large number of $P$. mirabilis obtained exclusively from humans and companion animals with UTIs.

In this study, the $P$. mirabilis clonal relatedness was accessed only by PFGE analysis and not also by Multi Locus Sequencing Typing because the latter isn't available for this bacterial specie. Future studies using whole genome sequencing could help to overcome this limitation and further contribute to understand the risk of uropathogenic $P$. mirabilis animal-human transfer.

Studies conducted on E. coli, have found that companion animals and humans may share uropathogenic strains within the same household (Johnson and Clabots, 2006). Similar studies should be conducted regarding $P$. mirabilis to fully evaluate the risk of animal-human transfer during UTI. Nevertheless, considering the clonal relatedness between $P$. mirabilis from companion animals and humans in this study, the role of companion animals in the dissemination of uropathogenic resistant $P$. mirabilis should not be neglected. The route of transfer (human to animal and vice versa) is also unknown and warrants attention in future studies. The lack of hygiene practices has been pointed as a factor for dissemination of P. mirabilis (Drzewiecka, 2016) and therefore should be considered when handling companion animals.

\section{Conclusion}

This study shows that UTI in companion animals and humans may be caused by closely related $P$. mirabilis strains and that $P$. mirabilis from both origins have common antimicrobial resistant and virulence genes. Taken together, these results underline the possible zoonotic nature of $P$. mirabilis causing UTIs in companion animals, which has important public-health implications.

Pending additional research, companion animals might be regarded as possible sources of uropathogenic $P$. mirabilis to humans and other companion animals living in close contact.

\section{Funding}

This work was supported by FEDER funds through the Programa Operacional Factores de Competitividade - COMPETE and by National funds through the FCT - Fundação para a Ciência e a Tecnologia [UID/ CVT/00276/2013] and by the Joint Programming Initiative on Antimicrobial Resistance [JPIAMR/0002/2016 (PET-Risk Consortium)]. Adriana Belas and Cátia Marques hold PhD grant SFRH/ BD/113142/2015 and SFRH/BD/77886/2011, respectively 


\section{Conflict of interests statement}

None of the authors has any financial or personal relationships that could inappropriately influence or bias the content of the paper.

\section{Declarations of interest}

Nothing to declare.

\section{Acknowledgments}

We gratefully acknowledge Molly Freeman, PhD from the Centers for Disease Control and Prevention, USA, for the help regarding optimization of the Pulse Field Gel Electrophoresis typing of $P$. mirabilis strains.

\section{Appendix A. Supplementary data}

Supplementary material related to this article can be found, in the online version, at doi:https://doi.org/10.1016/j.vetmic.2018.10.015.

\section{References}

Antunes, P., Machado, J., Sousa, J.C., Peixe, L., 2005. Dissemination of sulfonamide re sistance genes (sul1, sul2, and sul3) in Portuguese Salmonella enterica strains and relation with integrons. Antimicrob. Agents Chemother. 49, 836-839.

Aogáin, M.M., Rogers, T.R., Crowley, B., 2016. Identification of emergent blaCMY-2carrying Proteus mirabilis lineages by whole-genome sequencing. New Microbes New Infect. 9, 58-62.

Armbruster, C.E., Mobley, H.L., 2012. Merging mythology and morphology: the multifaceted lifestyle of Proteus mirabilis. Nat. Rev. Microbiol. 10, 743-754.

Bijlsma, I.G., van Dijk, L., Kusters, J.G., Gaastra, W., 1995. Nucleotide sequences of two fimbrial major subunit genes, $p m p A$ and $u c a A$, from canine-uropathogenic Proteus mirabilis strains. Microbiology 141, 1349-1357.

Cavaco, L.M., Hasman, H., Xia, S., Aarestrup, F.M., 2009. qnrD, a novel gene conferring transferable quinolone resistance in Salmonella enterica Serovar Kentucky and Bovismorbificans strains of human origin. Antimicrob. Agents Chemother. 53, 603-608.

Cestari, S.E., Ludovico, M.S., Martins, F.H., da Rocha, S.P., Elias, W.P., Pelayo, J.S., 2013. Molecular detection of HpmA and HlyA hemolysin of uropathogenic Proteus mirabilis. Curr. Microbiol. 67, 703-707.

CLSI, 2017. Performance Standards for Antimicrobial Susceptibility Testing. CLSI Supplement M100S-S27, 26th ed. Clinical and Laboratory Standards Institute, Wayne, PA, USA 2017.

CLSI, 2018. Performance Standards for Antimicrobial Disk and Dilution Susceptibility Tests for Bacteria Isolated From Animals. CLSI Supplement VET08, 4th ed. Clinical and Laboratory Standards Institute, Wayne, PA, USA 2018.

Costa, T., Linhares, I., Ferreira, R., Neves, J., Almeida, A., 2018. Frequency and antibiotic resistance of bacteria implicated in community urinary tract infections in North Aveiro between 2011 and 2014. Microb. Drug Resist. 24, 493-504.

de Jong, A., Muggeo, A., El Garch, F., Moyaert, H., de Champs, C., Guillard, T., 2018. Characterization of quinolone resistance mechanisms in Enterobacteriaceae isolated from companion animals in Europe (ComPath II study). Vet. Microbiol. 216, 159-167.

D’Andrea, M.M., Literacka, E., Zioga, A., Giani, T., Baraniak, A., Fiett, J., Sadowy, E., Tassios, P.T., Rossolini, G.M., Gniadkowski, M., Miriagou, V., 2011. Evolution and spread of a multidrug-resistant Proteus mirabilis clone with chromosomal AmpC-type cephalosporinases in Europe. Antimicrob. Agents Chemother. 55, 2735-2742.

Di Pilato, V., Chiarelli, A., Boinett, C.J., Riccobono, E., Harris, S.R., D’Andrea, M.M., Thomson, N.R., Rossolini, G.M., Giani, T., 2016. Complete genome sequence of the first KPC-type carbapenemase-positive Proteus mirabilis strain from a bloodstream infection. Genome Announc. 4 e00607-16.

Drzewiecka, D., 2016. Significance and roles of Proteus spp. bacteria in natural environments. Microb. Ecol. 72, 741-758.

Frana, T.S., Carlson, S.A., Griffith, R.W., 2001. Relative distribution and conservation of genes encoding aminoglycoside-modifying enzymes in Salmonella enterica Serotype Typhimurium phage type DT104. Appl. Environ. Microbiol. 67, 445-448.

Gaastra, W., van Oosterom, R.A., Pieters, E.W., Bergmans, H.E., van Dijk, L., Agnes, A., ter Huurne, H.M., 1996. Isolation and characterisation of dog uropathogenic Proteus mirabilis strains. Vet. Microbiol. 48, 57-71.

Guerra, B., Soto, S.M., Argüelles, J.M., Mendoza, M.C., 2001. Multidrug resistance is mediated by large plasmids carrying a Class 1 integron in the emergent Salmonella enterica Serotype [4,5,12:i: - ]. Antimicrob. Agents Chemother. 45, 1305-1308.

Hall, R.M., 2010. Salmonella genomic islands and antibiotic resistance in Salmonella enterica. Future Microbiol. 5, 1525-1538.

Harada, K., Niina, A., Shimizu, T., Mukai, Y., Kuwajima, K., Miyamoto, T., Kataoka, Y., 2014. Phenotypic and molecular characterization of antimicrobial resistance in Proteus mirabilis isolates from dogs. J. Med. Microbiol. 63, 1561-1567.

Hernández, J.R., Martínez-Martínez, L., Pascual, A., Suárez, A.I., Perea, E.J., 2000. Trends in the susceptibilities of Proteus mirabilis isolates to quinolones. J. Antimicrob. Chemother. 45, 407-408.

Jacoby, G.A., Strahilevitz, J., Hooper, D.C., 2014. Plasmid-mediated quinolone resistance. Microbiol. Spectr. 2. https://doi.org/10.1128/microbiolspec.PLAS-0006-2013.

Johnson, J.R., Clabots, C., 2006. Sharing of virulent Escherichia coli clones among household members of a woman with acute cystitis. Clin. Infect. Dis. 43, e101-e108.

Magiorakos, A.P., Srinivasan, A., Carey, R.B., Carmeli, Y., Falagas, M.E., Giske, C.G., Harbarth, S., Hindler, J.F., Kahlmeter, G., Olsson-Liljequist, B., Paterson, D.L., Rice, L.B., Stelling, J., Struelens, M.J., Vatopoulos, A., Weber, J.T., Monnet, D.L., 2012. Multidrug-resistant, extensively drug-resistant and pandrug-resistant bacteria: an international expert proposal for interim standard definitions for acquired resistance. Clin. Microbiol. Infect. 18, 268-281.

Marques, C., Belas, A., Franco, A., Aboim, C., Gama, L.T., Pomba, C., 2017. Increase in antimicrobial resistance and emergence of major international high-risk clonal lineages in dogs and cats with urinary tract infection: 16 year retrospective study. J. Antimicrob. Chemother. 73, 377-384.

Mathur, S., Sabbuba, N.A., Suller, M.T., Stickler, D.J., Feneley, R.C., 2008. Genotyping of urinary and fecal Proteus mirabilis isolates from individuals with long-term urinary catheters. Eur. J. Clin. Microbiol. Infect. Dis. 24, 643-644.

Maynard, C., Fairbrother, J.M., Bekal, S., Sanschagrin, F., Levesque, R.C., Brousseau, R., Masson, L., Larivière, S., Harel, J., 2003. Antimicrobial resistance genes in enterotoxigenic Escherichia coli O149:K91 isolates obtained over a 23-year period from pigs. Antimicrob. Agents Chemother. 47, 3214-3221.

Moyaert, H., Morrissey, I., de Jong, A., El Garch, F., Klein, U., Ludwig, C., Thiry, J., Youala, M., 2017. Antimicrobial susceptibility monitoring of bacterial pathogens isolated from urinary tract infections in dogs and cats across Europe: ComPath results. Microb. Drug Resist. 23, 391-403.

Perreten, V., Boerlin, P., 2003. A new sulfonamide resistance gene (sul3) in Escherichia coli is widespread in the pig population of Switzerland. Antimicrob. Agents Chemother. 47, 1169-1172.

Pomba, C., Mendonça, N., Costa, M., Louro, D., Baptista, B., Ferreira, M., Correia, J.D., Caniça, M., 2006. Improved multiplex PCR method for the rapid detection of betalactamase genes in Escherichia coli of animal origin. Diagn. Microbiol. Infect. Dis. 56, 103-106.

Pomba, C., Rantala, M., Greko, C., Baptiste, K.E., Catry, B., van Duijkeren, E., Mateus, A., Moreno, M.A., Pyörälä, S., Ružauskas, M., Sanders, P., Teale, C., Threlfall, E.J. Kunsagi, Z., Torren-Edo, J., Jukes, H., Törneke, K., 2017. Public health risk of antimicrobial resistance transfer from companion animals. J. Antimicrob. Chemother. 72, 957-968.

Razavi, M., Marathe, N.P., Gillings, M.R., Flach, C.F., Kristiansson, E., Joakim Larsson, D.G., 2017. Discovery of the fourth mobile sulfonamide resistance gene. Microbiome $5,160$.

Robicsek, A., Strahilevitz, J., Jacoby, G.A., Macielag, M., Abbanat, D., Park, C.H., Bush, K., Hooper, D.C., 2006a. Fluoroquinolone-modifying enzyme: a new adaptation of a common aminoglycoside acetyltransferase. Nat. Med. 12, 83-88.

Robicsek, A., Strahilevitz, J., Sahm, D.F., Jacoby, G.A., Hooper, D.C., 2006b. qnrprevalence in ceftazidime-resistant Enterobacteriaceae isolates from the United States. Antimicrob. Agents Chemother. 50, 2872-2874.

Sabbuba, N.A., Mahenthiralingam, E., Stickler, D.J., 2003. Molecular epidemiology of Proteus mirabilis infections of the catheterized urinary tract. J. Clin. Microbiol. 41, 4961-4965.

Schultz, E., Baucheron, S., Lupo, A., Saras, E., Cloeckaert, A., Doublet, B., Madec, J.Y. Haenni, M., 2017a. Complete sequences of qnrD-carrying plasmids inProteus spp. collected from French animals. J. Glob. Antimicrob. Resist. 10, 306-307.

Schultz, E., Cloeckaert, A., Doublet, B., Madec, J.-Y., Haenni, M., 2017b. Detection of SGI1/PGI1 elements and resistance to extended-spectrum cephalosporins in Proteae of animal origin in France. Front. Microbiol. 8, 32

Schultz, E., Haenni, M., Mereghetti, L., Siebor, E., Neuwirth, C., Madec, J.Y., Cloeckaert, A., Doublet, B., 2015. Survey of multidrug resistance integrative mobilizable elements SGI1 and PGI1 in Proteus mirabilis in humans and dogs in France, 2010-13. J. Antimicrob. Chemother. 70, 2543-2546.

Sáenz, Y., Briñas, L., Domínguez, E., Ruiz, J., Zarazaga, M., Vila, J., Torres, C., 2004. Mechanisms of resistance in multiple-antibiotic-resistant Escherichia coli strains of human, animal, and food origins. Antimicrob. Agents Chemother. 48, 3996-4001.

SFM, 2010. Comité de l'antibiogramme de la Société Française de Microbiologie: Recommandations 2010. Société Française de Microbiologie, Paris, France.

Sosa, V., Schlapp, G., Zunino, P., 2006. Proteus mirabilis isolates of different origins do not show correlation with virulence attributes and can colonize the urinary tract of mice. Microbiology 152, 2149-2157.

Wang, M., Guo, Q., Xu, X., Wang, X., Ye, X., Wu, S., Hooper, D.C., Wang, M., 2009. New plasmid-mediated quinolone resistance gene, $q n r C$, found in a clinical isolate of Proteus mirabilis. Antimicrob. Agents Chemother. 53, 1892-1897.

Weigel, L.M., Anderson, G.J., Tenover, F.C., 2002. DNA gyrase and topoisomerase IV mutations associated with fluoroquinolone resistance in Proteus mirabilis. Antimicrob. Agents Chemother. 46, 2582-2587. 